\title{
Síntese, caracterização magnética e elétrica da ferrita de aluminato de cobre
}

\author{
Synthesis, characterization magnetic and electrical of aluminate copper ferrite \\ Síntesis, caracterización magnética y eléctrica de ferrita de cobre aluminato
}

Recebido: 17/06/2021 | Revisado: 25/06/2021 | Aceito: 28/06/2021 | Publicado: 13/07/2021

\author{
Vander Alkmin dos Santos Ribeiro \\ ORCID: https://orcid.org/0000-0003-0260-2454 \\ Centro Universitário de Itajubá, Brasil \\ E-mail: vanderalkmin@gmail.com \\ Valesca Donizete de Oliveira \\ ORCID: https://orcid.org/0000-0002-1781-8605 \\ Universidade Federal de Itajubá, Brasil \\ E-mail: valescadooliveira@unifei.edu.br \\ Rero Marques Rubinguer \\ ORCID: https://orcid.org/0000-0003-1718-9658 \\ Universidade Federal de Itajubá, Brasil \\ E-mail: rero@unifei.edu.br \\ Adhimar Flávio Oliveira \\ ORCID: https://orcid.org/0000-0003-2586-7359 \\ Universidade Federal de Itajubá, Brasil \\ E-mail: adhimarflavio@unifei.edu.br \\ Claudiney Sales de Pereira Mendonça \\ ORCID: https://orcid.org/0000-0002-2271-9900 \\ Universidade Federal de Itajubá, Brasil \\ E-mail: claudiney.mendonca@educacao.mg.gov.br
}

\begin{abstract}
Resumo
Atualmente, as ferritas têm sido tema de muitas pesquisas devido as suas vantagens e propriedades que podem ser facilmente manipuladas, sendo assim de grande interesse tecnológico e científico. Para tanto, a distribuição dos cátions e interações magnéticas destacam um papel importante nestes materiais e, portanto, têm sua importância científica. As relações entre a composição química, estrutura cristalina, comportamento magnético e elétrico foram investigadas em ferritas de aluminato de cobre. As ferritas $\mathrm{CuAl}_{\mathrm{X}} \mathrm{Fe}_{2-\mathrm{X}} \mathrm{O}_{4}$, onde $\mathrm{x}=0,0 ; 0,5 ; 1,0$ e 1,5 foram obtidas pelo método convencional de cerâmicas, reação estado-sólido entre os óxidos de ferro, alumínio e cobre. A mistura de óxidos foram pré-sinterizadas por 24 horas a $800^{\circ} \mathrm{C}$ e depois sinterizadas a $1100^{\circ} \mathrm{C}$ durante $8 \mathrm{~h}$. As propriedades magnéticas foram medidas por um magnetômetro de amostra vibrante e determinadas a partir do gráfico de histerese, observando-se que possui um comportamento de um material magnético moderado devido ao perfil da curva de magnetização e valores de coercividade $(\sim 223 \mathrm{kA} / \mathrm{m})$. A condutividade elétrica das pastilhas foi obtida a partir de características de tensão pela corrente em função da temperatura. A dependência da condutividade elétrica com a temperatura das ferritas de aluminato de cobre com diferentes composições apresentou um comportamento semicondutor e com o aumento da resistividade do material com o aumento do teor de alumínio ocorre devido à sua propriedade condutora. Também se observou que a magnetização de saturação diminui com o aumento da concentração de alumínio, apresentando comportamento de um material paramagnético e mole.
\end{abstract}

Palavras-chave: Ferritas; Propriedades magnéticas; Condutividade elétrica; Energia de ativação.

\begin{abstract}
Currently, ferrites have been the subject of much research due to their advantages and properties that can be easily manipulated, thus being of great technological and scientific interest. Therefore, the distribution of cations and magnetic interactions highlight an important role in these materials and, therefore, have their scientific importance. The relationships between chemical composition, crystal structure, magnetic and electrical behavior were investigated in copper aluminum ferrites. The $\mathrm{CuAl}_{\mathrm{X}} \mathrm{Fe}_{2-\mathrm{x}} \mathrm{O}_{4}$ ferrites, where $\mathrm{x}=0.0 ; 0.5 ; 1.0$, and 1.5 were obtained by the conventional ceramic method, the solid-state reaction between iron, aluminum, and copper oxides. The oxide mixture was pre-sintered for 24 hours at $800^{\circ} \mathrm{C}$ and then sintered at $1100^{\circ} \mathrm{C}$ for 8 hours. The magnetic properties were measured by a vibrating sample magnetometer and determined from the hysteresis graph, noting that it has a moderate magnetic material behavior due to the magnetization curve profile and coercivity values $(\sim 223 \mathrm{kA} / \mathrm{m})$. The electrical conductivity of the pellets was obtained from voltage-by-current characteristics as a function of temperature. The electrical conductivity of the pellets was obtained from voltage-by-current characteristics as a function of temperature. The dependence of electrical conductivity with the temperature of copper aluminate ferrites with different compositions presented a semiconductor behavior and with the increase of the material resistivity with the increase of
\end{abstract}


the aluminum content, it occurs due to its conductive property. It was also observed that the saturation magnetization decreases with increasing aluminum concentration, showing the behavior of a soft paramagnetic material.

Keywords: Ferrites; Magnetic properties; Electric conductivity; Activation energy.

\section{Resumen}

En la actualidad, las ferritas han sido objeto de mucha investigación debido a sus ventajas y propiedades de fácil manipulación, siendo así de gran interés tecnológico y científico. Por tanto, la distribución de cationes y las interacciones magnéticas destacan un papel importante en estos materiales y, por tanto, tienen su importancia científica. Se investigaron las relaciones entre la composición química, la estructura cristalina, el comportamiento magnético y eléctrico en ferritas de cobre y aluminio. Las ferritas CuAlXFe2-XO4, donde x = 0.0; 0,5;1.0 y $1.5 \mathrm{se}$ obtuvieron por el método cerámico convencional, la reacción en estado sólido entre óxidos de hierro, aluminio y cobre. La mezcla de óxidos se pre-sinterizó durante 24 horas a $800{ }^{\circ} \mathrm{C}$ y luego se sinterizó a $1100{ }^{\circ} \mathrm{C}$ durante 8 horas. Las propiedades magnéticas se midieron con un magnetómetro de muestra vibrante y se determinaron a partir del gráfico de histéresis, notando que tiene un comportamiento de material magnético moderado debido al perfil de la curva de magnetización y los valores de coercitividad $(\sim 223 \mathrm{kA} / \mathrm{m})$. La conductividad eléctrica de los gránulos se obtuvo a partir de las características de voltaje por corriente en función de la temperatura. La conductividad eléctrica de los gránulos se obtuvo a partir de las características voltaje por corriente en función de la temperatura. La dependencia de la conductividad eléctrica con la temperatura de las ferritas de aluminato de cobre con diferentes composiciones mostró un comportamiento semiconductor y con el aumento de la resistividad del material con el aumento del contenido de aluminio, se produce debido a su propiedad conductora. También se observó que la magnetización de saturación disminuye al aumentar la concentración de aluminio, mostrando el comportamiento de un material paramagnético y blando.

Palabras clave: Ferritas; Propiedades magnéticas; Conductividad eléctrica; Energía de activación.

\section{Introdução}

A Ferrita de cobre-alumínio $\mathrm{CuFe}_{2-\mathrm{x}} \mathrm{Al}_{\mathrm{x}} \mathrm{O}_{4}$ pertence a uma grande classe de compostos que têm a fórmula geral $\mathrm{A}^{+2}$ $\mathrm{B}_{2}{ }^{+3} \mathrm{O}_{4}{ }^{-2}$ e apresenta uma estrutura cristalina tipo espinélio. As ferritas de espinélio têm sido usadas como materiais eletrônicos e são adequadas para o mercado de sensores de gás. Elas são simples, de baixo custo, e em comparação com outros sensores de gás têm estrutura e versatilidade de composição. Os mecanismos de detecção de uma gama de gases e umidade são explicados pelo mecanismo de condução através de sensibilização eletrônica e química que ambos aumentam a condutividade (Sutka \& Gross, 2016).

Estes materiais apresentam estrutura cristalina cúbica. A rede cúbica é constituída por células unitárias que contém um total de 56 íons, sendo 32 íons de oxigênio, 16 cátions octaédricos e 8 cátions tetraédricos para o espinélio normal. Enquanto, a estrutura cúbica de espinelio inverso apresenta a mesma quantidade de 56 íons com arranjo de 8 cátions divalente em sítios tetraédricos e 16 cátions trivalentes em 8 sítios tetraédricos e 8 octaédricos. As ferritas espinélios são representadas pela fórmula $\mathrm{MeFe}_{2} \mathrm{O}_{4}$, no qual Me designa-se um metal divalente. Ferritas tipo espinélios invertidos que apresentam cátions bivalentes em coordenação tetraédrica e cátions trivalentes $\left(\mathrm{Fe}^{+3}\right)$ ocupando posições intersticiais tetraédricas e octaédricas simultaneamente (Smit e Wijn, 1959; Skołyszewska et. Al. 2003; Chae et. Al. 2013).

A substituição parcial de íons $\mathrm{Al}^{+3}$ por $\mathrm{Fe}^{+3}$ em ferritas de espinélio podem apresentar alguns efeitos benéficos em propriedades dielétricas, elétricas, magnéticas e elásticas. As propriedades magnéticas de ferritas espinélio são drasticamente influenciadas pelo tipo de cátions, assim como, a sua distribuição, em qualquer das sub-redes do material investigado. $\mathrm{O}$ sistema da ferrita de $\mathrm{CuFe}_{2-\mathrm{x}} \mathrm{Al}_{\mathrm{x}} \mathrm{O}_{4}$ sugere que o íon de $\mathrm{Cu}^{+2}$ prefere ocupar o sitío octaédrico (B) e que íons $\mathrm{Al}^{+3} \mathrm{e} \mathrm{Fe}^{+3}$ podem ocupar tantos sítios tetraédricos e octaédricos (A e B). A dopagem com íons diamagnéticos em ferritas de espinélio enfraquece o acoplamento magnético no interior da estrutura, que podem alterar as propriedades magnéticas (Raghavender et al. 2013).

Um dos efeitos da dopagem com cobre na estrutura de espinélio é a distorção tetragonal que ocorre no parâmetro de rede na estrutura cúbica da célula original. Isto altera o sistema que passa de espinélio cúbico para o sistema tetragonal, ou seja, a célula unitária do espinélio pode sofrer um discreto alongamento em uma das arestas, quando da inclusão de certa quantidade de cobre. Isto ocorre em virtude do chamado efeito Jahn-Teller que é a transição de fase cúbica para tetragonal 
ocorrendo um crescimento do eixo $a$ da estrutura cúbica em um eixo mais longo $c$, com parâmetros de célula unitária $a=5,882 \AA$ e $c=8,337 \AA$ (Ribeiro et al. 2016; Tanaka et al. 1997; Dunitz e Orgel, 1957; Sugimoto, 1977).

Segundo Surashe (2020), o efeito Jahn-Teller provoca a distorção do espinélio da ferrita de cobre à temperatura ambiente provocando assim uma distorção para a simetria tetragonal, fato que depende apenas da distribuição do íon de cobre nos sítios. As propriedades estruturais e elétricas da ferrita de cobre foram investigadas por difração de raios-x e estudos de resistividade DC. Os dados de XRD à temperatura ambiente revelaram que a formação de estrutura tetragonal de $\left(\mathrm{CuFe}_{2} \mathrm{O}_{4}\right) \mathrm{e}$ o parametro de rede (a) foi encontrada no valor de $8,380 \AA$ Á.

Segundo Raghavender (2012), a dopagem de alumínio na ferrita de cobre causa mudanças significativas nas propriedades estruturais e magnéticas. Através da análise dos espectros do raios-X mostraram que o $\mathrm{Cu}^{2+} \mathrm{e} \mathrm{Al}^{3+}$ ocupavam preferencialmente os sítios octaédricos, enquanto que a concentração de $\mathrm{Fe}^{3+}$ ocupava os sítios tetraédricos. Esses resultados mostraram que conseguentemente ocorreu mudanças na estrutura e na composição para permite o ajuste das propriedades magnéticas. Também foi observado uma diminuição na magnetização de saturação, a remanência e o campo coercitivo com o aumento da concentração de $\mathrm{Al}^{3+}$.

O objetivo do trabalho é apresentar a síntese da ferrita de aluminato de cobre, suas propriedades magnéticas, elétricas e estrutural. A influência do cobre sobre algumas composições de ferritas tem sido bastante investigada por vários pesquisadores, ao longo das últimas décadas, com o intuito de melhorar algumas propriedades e características destes materiais. Por outro lado, as ferritas dopadas com cobre têm despertado interesse da indústria eletrônica devido às boas propriedades elétricas e magnéticas em dispositivos que atuam em altas freqüências (Ribeiro et al. 2019).

Dentre os resultados apresentados podemos salientar que dependência da condutividade elétrica com a temperatura das ferritas de aluminato de cobre com diferentes composições apresentou um comportamento semicondutor e das medidas magnéticas possui um comportamento de um material magnético moderado.

\section{Materiais e Métodos}

Uma série de ferritas de $\mathrm{CuAl}_{\mathrm{XFe}} \mathrm{Fe}_{2} \mathrm{O}_{4}$, onde $\mathrm{x}=0,0 ; 0,5 ; 1,0$ e 1,5, foram produzidas via metalurgia do pó (Oliveira et al. 2016) utilizando-se os óxidos de cobre, ferro e de alumínio de elevada de pureza (99,9\%). Os pós nas estequiometrias definidas foram compactados uniaxialmente na forma de pastilhas $(\varnothing=8 \mathrm{~mm})$, utilizando uma pressão de $40 \mathrm{MPa}$.

As amostras foram sinterizadas em um forno com atmosfera ambiente, por um período de tempo de 32 horas, onde permaneceu à $800^{\circ} \mathrm{C}$ por 24 horas e $1100^{\circ} \mathrm{C}$ por $8 \mathrm{~h}$.

As medidas de difração de raios $\mathrm{X}$ foram realizadas, com as amostras na forma de pó, utilizando-se um difratômetro Panalytical modelo X-Pert Powder com varredura (20) de 15 a 85 graus, passo de 0,02 graus e tempo de contagem de 1 segundo/passo.

A caracterização magnética foi realizada em um Magnetômetro de Amostra Vibrante da marca Lakeshore, modelo 7404 a temperatura ambiente. Os parâmetros magnéticos: campo coercivo $\left(H_{c}\right)$, magnetização de saturação $\left(M_{s}\right)$ e magnetização remanente foram determinados através das curvas de magnetização com um campo aplicado de $14 \mathrm{kG}$ (Ribeiro et al. 2016).

A condutividade elétrica em corrente contínua (DC) das pastilhas cilíndricas com $8 \mathrm{~mm}$ de diâmetro foi avaliada pelo método de dois pontos, utilizando um forno com controle de atmosfera e uma Source-Meter 2600 da Keithley (Ribeiro et al. 2021). O comportamento da resistividade elétrica em função da temperatura foi avaliado para um gradiente de temperatura de $5 \mathrm{~K} / \mathrm{min}$ ao longo do ensaio com temperatura até $350^{\circ} \mathrm{C}$ sob atmosfera de 1 atm de nitrogênio seco. 


\section{Resultados e Discussão}

A Figura 1 apresenta os difratogramas das amostras $\mathrm{CuAl}_{\mathrm{X}} \mathrm{Fe}_{2-\mathrm{X}} \mathrm{O}_{4}$ com diferentes composições. As amostras com estequiometria $\mathrm{x}=0$ são formadas pelas fases espinélio de cobre com estrutruras cúbica (JCPDS 56-591-0029) e tetragonal (JCPDS 96-901-1013). As amostras com x = 0,5 são formadas exclusivamente pela fase espinélio de cobre cúbico. As amostras com $\mathrm{x}=1,0$ são formadas pelas fases espinélio de cobre cúbico e aluminato de cobre. As amostras com $\mathrm{x}=1,5$ é formada exclusivamente pela fase aluminato de cobre.

Figura 1. Difração de raios- $\mathrm{X}$ da ferrita de $\mathrm{CuAl}_{\mathrm{X}} \mathrm{Fe}_{2-\mathrm{X}} \mathrm{O}_{4}$

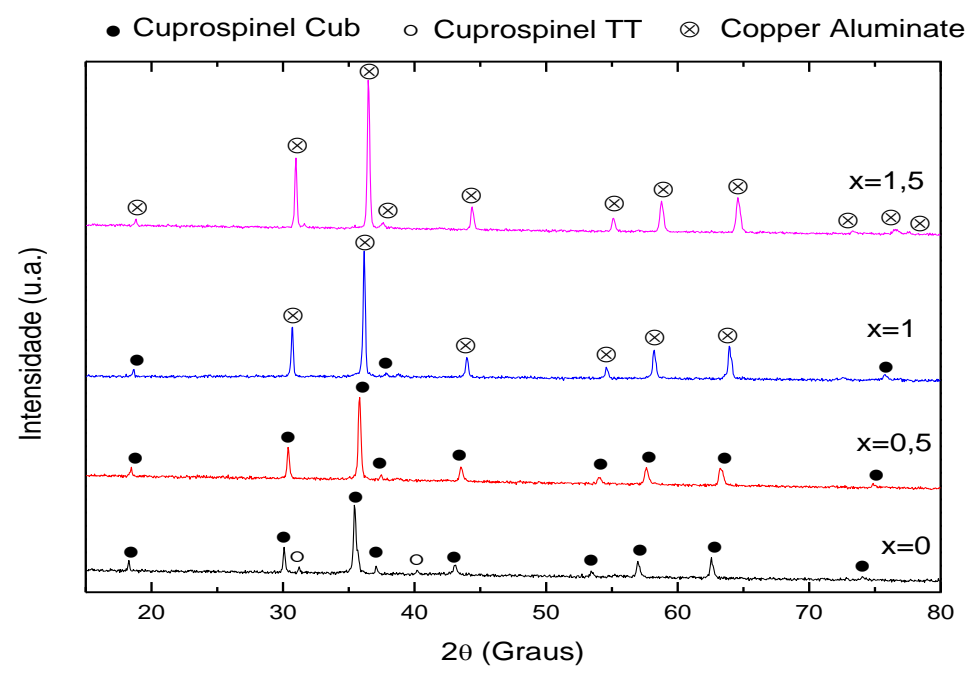

Fonte: Autores (2021).

A Figura 2 representa a magnetização $(\mathrm{M})$ em função do campo externo aplicado $(\mathrm{H})$ à temperatura ambiente. Os valores de magnetização de saturação $\left(M_{s}\right)$, coercividade $\left(H_{c}\right)$ e magnetização remanente $\left(M_{R}\right)$ são obtidos a partir das curvas de histerese e estão representados na Tabela 1. Observou-se que a magnetização de saturação diminui com o aumento da concentração de alumínio, apresentando comportamento de um material paramagnético e mole. A adição de íons de $\mathrm{Al}^{3+}$, que tem forte preferência para os sítios octaédricos deve exibir a diminuição da magnetização de saturação porque os íons não são magnéticos.

Foi observado como aumento da quantidade de $\mathrm{Al}^{3+}$, o momento magnético de célula unitária diminui. Conclui-se que os ângulos dos cátions aumentam com o aumento de $\mathrm{Al}^{3+}$, o que indica o favorecimento de momento de rotação triangular nos sítios octaédricos levando à redução na interação de subrede. A remoção dos íons de $\mathrm{Fe}^{3+}$ magnético da subrede magnética e substituição por íons de $\mathrm{Al}^{3+}$, que é considerado não magnético, enfraquecem as interações de troca, que tendem a alinhar os dipolos antiparalelos (Gabal et al,. 2013). Este material apresenta o comportamento de um material magneticamente moderado, a área relativa dentro do ciclo de histerese deve ser pequena, sendo caracteristicamente fina e estreita; sendo utilizados em dispositivos que estão sujeitos a campos magnéticos alternados, nos quais as perdas de energia devem ser baixas (Mathew e Juang 2007). 
Figura 2. Curva de Magnetização versus campo magnético aplicado em temperatura ambiente de $\mathrm{CuAl}_{\mathrm{X}} \mathrm{Fe}_{2-\mathrm{X}} \mathrm{O}_{4}$.

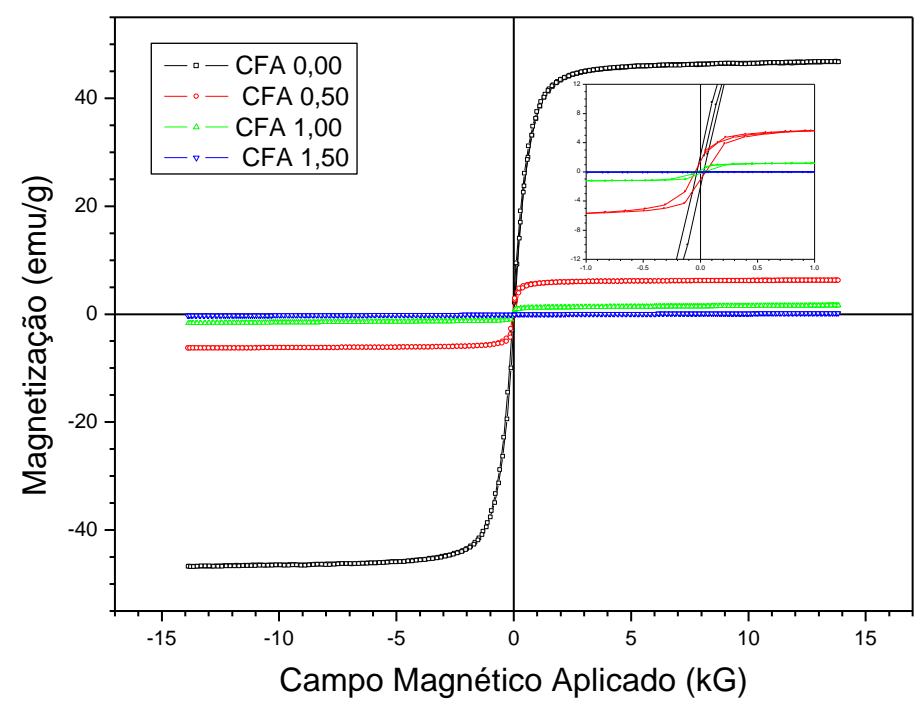

Fonte: Autores (2021).

Tabela 1. Remanescência $(\mathrm{Br})$, coercividade $(\mathrm{Hc})$, magnetização de saturação $(\mathrm{Ms})$ à temperatura ambiente para as ferritas de $\mathrm{CuAl}_{\mathrm{X}} \mathrm{Fe}_{2-\mathrm{X}} \mathrm{O}_{4}$.

\begin{tabular}{|l|l|l|l|}
\hline \multicolumn{1}{|c|}{ Amostras } & $\mathrm{M}_{\mathrm{S}}(\mathrm{emu} / \mathrm{g})$ & $\mathrm{M}_{\mathrm{R}}(\mathrm{emu} / \mathrm{g})$ & $\mathrm{H}_{\mathrm{C}}(\mathrm{G})$ \\
\hline $\mathrm{CuFe}_{2} \mathrm{O}_{4}$ & 42,84 & 1,95 & 19,00 \\
\hline $\mathrm{CuAl}_{0,5} \mathrm{Fe}_{1,5} \mathrm{O}_{4}$ & 5,94 & 1,27 & 49,51 \\
\hline $\mathrm{CuAl}_{1,0} \mathrm{Fe}_{1,0} \mathrm{O}_{4}$ & 1,50 & 0,19 & 31,19 \\
\hline $\mathrm{CuAl}_{1,5} \mathrm{Fe}_{0,5} \mathrm{O}_{4}$ & 0,02 & 0,00069 & 91,69 \\
\hline
\end{tabular}

Fonte: Autores (2021).

Na Figura 3 ilustra a dependência da resistividade elétrica em função da temperatura em diferentes concentrações das ferritas investigadas até $350^{\circ} \mathrm{C}$. A resistividade elétrica $(\rho)$ das ferritas foi calculada a partir das medidas de corrente para uma tensão aplicada constante usando a equação 1.

$$
\rho=\frac{V A}{i t}
$$

em que $i$ é a corrente que passa através da amostra em amperes, $V$ é a voltagem aplicada à amostra, em volts, $t$ é a espessura da amostra em centímetros e $A$ indica a área da amostra em $\mathrm{cm}^{2}$.

Observou-se na Figura 3 que todas as amostras apresentam uma característica isolante/semicondutora, i.e. com redução exponencial da resistividade com o aumento da temperatura. O valor da resistividade é proporcional à quantidade de Al na ferrita. Este resultado pode ser explicado considerando que os íons de $\mathrm{Al}^{3+}$ residem principalmente nos sítios octaédricos e que por isso eles impedem a troca de elétrons entre íons de $\mathrm{Fe}^{2+}$ e $\mathrm{Fe}^{3+}$ nos sítios octaédricos. Como o salto de elétrons entre $\mathrm{Fe}^{2}+\mathrm{e} \mathrm{Fe}^{3}+$ nos sítios octaédricos é o principal mecanismo de condução dessas ferritas, a substituição de íons de $\mathrm{Fe}^{2}+$ em virtude do aumento da concentração de $\mathrm{Al}^{3}+$ ocasiona o aumento da resistividade elétrica (Satar, 2004).

O valor da resistividade de uma ferrita como as desenvolvidas nesse estudo é influenciado por vários fatores como: temperatura de sinterização, microestrutura, porosidade, área de contorno de grão, bem como a conversão dos íons trivalentes de $\mathrm{Fe}^{3+}$ para o estado $\mathrm{Fe}^{2+}$. Quanto menor o tamanho do grão, maior será o contorno entre os grãos. Assim, como os contornos 
entre os grãos geralmente são isolantes, grãos menores implicam em resistividade mais elevada. Um aumento da temperatura de sinterização resulta em maior densidade e crescimento dos grãos, que diminui a porosidade e o número de contorno entre os grãos e, por sua vez leva a uma resistividade menor. E já que os poros não são condutores, a densificação é fator de diminuição da resistividade. Com o aumento temperatura de sinterização, a redução dos íons de $\mathrm{Fe}^{3+}$ para o $\mathrm{Fe}^{2+}$ ocorre nos sítios tetraédricos (A), pouco contribuem para a condução devido às distâncias maiores entre elas, mas a formação de íons de $\mathrm{Fe}^{2+}$ nos sítios octaédricos (B), dá origem à condução da ferrita devido ao movimento eletrônico entre os íons de $\mathrm{Fe}^{3+}$ e íons de $\mathrm{Fe}^{2+}$ espaçados na estrutura do espinélio e assim diminui a resistividade (Parfenov e Nazipov, 2002).

Figura 3. Resistividade elétrica em função da temperatura das ferritas de $\mathrm{CuAl}_{x} \mathrm{Fe}_{2-\mathrm{x}} \mathrm{O}_{4}$.

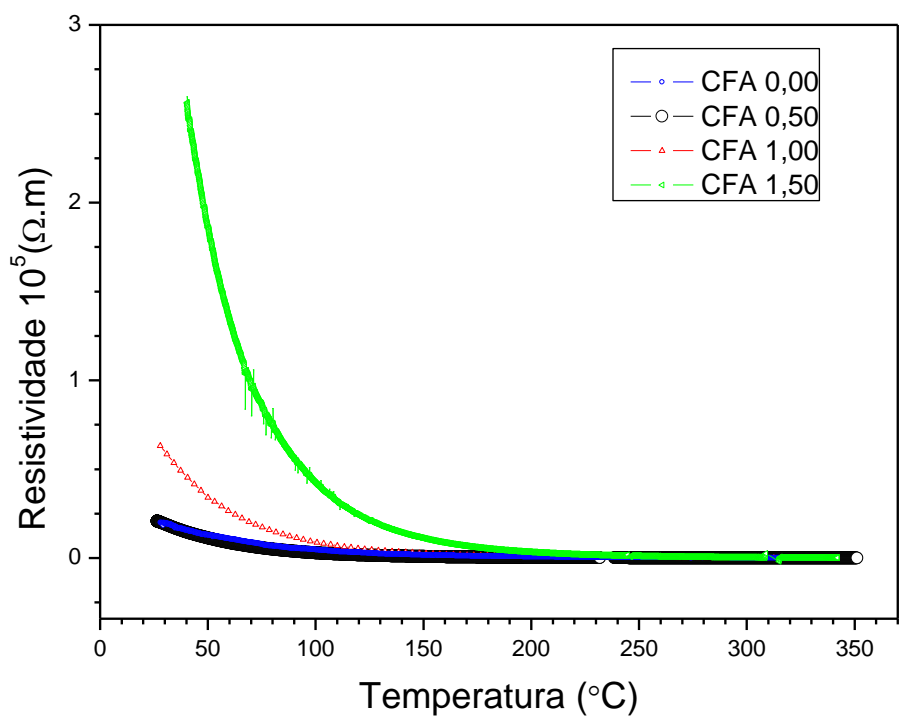

Fonte: Autores (2021).

Do ponto de vista do processo ou mecanismo de transporte de cargas podemos verificar que a condutividade é atribuída a saltos (i.e. hopping) de elétrons em estados eletrônicos ligados aos íons $\mathrm{Fe}^{2+}$ e $\mathrm{Fe}^{3+}$ que ocupam os sítios octaédricos. O salto corresponde a transformação $\mathrm{Fe}^{2+} \Rightarrow \mathrm{Fe}^{3+}+\mathrm{e}^{-}$. Jonker (1959) observou esse tipo de mecanismo de condução para uma ferrita de cobalto e, devido às similaridades entre as características dessas ferritas com as aqui apresentadas, espera-se que as ferritas de cobre e alumínio apresentem o mesmo processo, como será verificado. Por se tratar de um movimento entre estados eletrônicos mais localizados ou ligados esse tipo de transporte é conhecido como por saltos ou hopping em contraposição ao transporte em bandas com elétrons ou buracos quase livres que ocorremos semicondutores normais.

Nas ferritas, os portadores de carga não são livres para mover-se através da rede cristalina, mas podem ocorrer saltos entre íons. Assume-se que o número de portadores de carga (elétrons) que contribuem para esse processo seja da ordem do número de íons de $\mathrm{Fe}^{2+}$ e o número de estados disponíveis seja igual ao número de íons $\mathrm{Fe}^{3+}$.

$\mathrm{Na}$ presença de fônons ou vibrações da rede, a probabilidade da transferência de elétrons é mais alta devido ao fato dessas vibrações promoverem a aproximação dos íons em sítios condutores o que por sua vez aumenta a probabilidade de transferência de elétrons.

Assim, a condução é também induzida pelas vibrações da rede e, consequentemente, o deslocamento de portadores mostra a dependência da temperatura caracterizada pela energia de ativação. 
A diminuição da resistividade pode ser atribuída a vários fatores como: aumento da temperatura de sinterização, microestruturais, porosidade, área de contorno de grão, bem como a conversão dos íons trivalentes de $\mathrm{Fe}^{3+}$ para o estado $\mathrm{Fe}^{2+}$. Quanto menor o tamanho do grão, maior será o contorno entre os grãos. Assim, a resistividade é contribuída pelos contornos entre os grãos, considerados como isolantes. Um aumento da temperatura de sinterização resulta em maior densidade e crescimento dos grãos, que diminui a porosidade e o número de contorno entre os grãos. E já que os poros não são condutores, os portadores de carga enfrentam pequenas quantidades de poros diminuindo a resistividade. Com o aumento temperatura de sinterização, a redução dos íons de $\mathrm{Fe}^{3+}$ para o $\mathrm{Fe}^{2+}$ ocorre nos sítios tetraédricos (A), pouco contribuem para a condução devido às distâncias maiores entre elas, mas a formação de íons de $\mathrm{Fe}^{2+}$ nos sítios octaédricos (B), dá origem à condução da ferrita devido ao movimento eletrônico entre os íons de $\mathrm{Fe}^{3+}$ e íons de $\mathrm{Fe}^{2}+$ espaçados na estrutura do espinélio e assim diminui a resistividade.

A condutividade é atribuída a saltos de elétrons entre os íons $\mathrm{Fe}^{2+}$ e $\mathrm{Fe}^{3+}$ entre os sítios octaédricos, assim como $\mathrm{Fe}^{2+}$ $\Rightarrow \mathrm{Fe}^{3+}+\mathrm{e}^{-}$. Jonker observou que na ferrita de cobalto, que o transporte é diferente dos semicondutores normais. Nas ferritas, os portadores de carga não são livres para mover-se através da rede cristalina, mas podem ocorrer saltos entre íons (Rubinger et al. 2006).

Segundo Ribeiro et.al (2019), nas temperaturas mais elevadas, o número de modos de fônons disponível é tal que permite o salto entre quaisquer par de íons $\mathrm{Fe}^{2+}, \mathrm{Fe}^{3+}$ em sítios octaédricos. Dessa forma o transporte se dá com uma energia de ativação constante, indicado pela região linear no início (i.e. pequenos valores de 1/T nas abscissas) das curvas da Figura 4. Tal processo é conhecido na literatura como saltos entre vizinhos próximos ou nearest neighbor hopping e descrito pela equação:

$$
\sigma=\sigma_{0} \exp \left(\frac{-\Delta E}{k T}\right)
$$

em que $k$ é a constante de Boltzmann, $\Delta E$ representa a energia de ativação e $T$ é a temperatura absoluta em Kelvin.

$\mathrm{Na}$ Figura 4, nas temperaturas mais baixas, i.e. 1/T crescente, ocorre uma transição de linear para sublinear. Tal efeito deve-se a condução ocorrer em regime de saltos de alcance variável. Nessa situação, o número de modos de fônons disponível torna-se menor que a quantidade de estados acessíveis em pares de íons $\mathrm{Fe}^{2+}, \mathrm{Fe}^{3+}$ em sítios octaédricos. Assim a faixa de energia disponível fica menor que $\Delta \mathrm{E}$ e, passa a depender da temperatura. 
Figura 4. Gráfico de Arrhenius das ferritas de $\mathrm{CuAl}_{\mathrm{x}} \mathrm{Fe}_{2-\mathrm{x}} \mathrm{O}_{4}$.

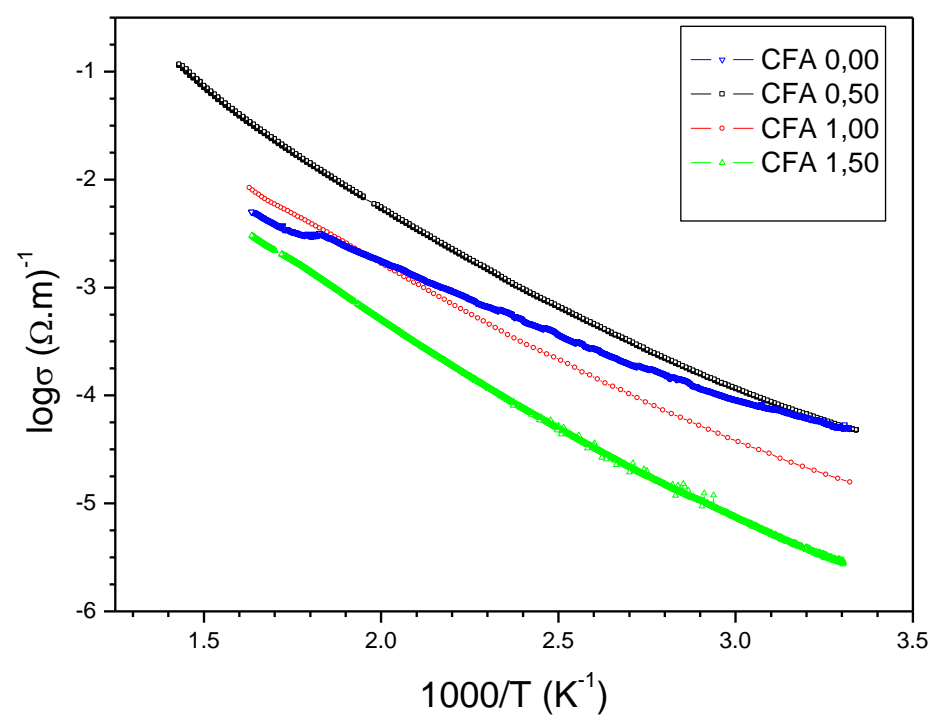

Fonte: Autores (2021).

Para se identificar a ocorrência do mecanismo de salto de alcance variável, bem como outras informações correlatas, utiliza-se um método de determinação de energia de ativação diferencial (Rubinger et al. 2006). Neste método considera-se o mecanismo de condução leva a uma condutividade do tipo:

$$
\sigma=\sigma_{0} \exp \left(\frac{-T_{0}}{T}\right)^{x}
$$

em que $T_{0}$ é o parâmetro de hopping e x o expoente que depende da dimensionalidade do transporte de cargas conforme descrito por (Rubinger et al., 2006).

Na Figura 5 ilustra a energia de ativação em função da temperatura para as amostras de ferrita de aluminato de cobre ferro determinadas pelo método descrito por (Rubinger et al., 2009). A partir dos dados apresentados na Figura 5 pode-se determinar a faixa de temperatura em que o mecanismo de salto variável, descrito pela equação (D) é dominante. A amostra de menor concentração parece ter o mecanismo de salto variável dominando até uma temperatura mais elevada, isto é, acima de $400^{\circ} \mathrm{C}$ e, apresentando maior energia de ativação. Acima desta temperatura ocorre o mecanismo de vizinhos próximos. As demais amostras com $\mathrm{x}=1,00$ e 1,50 fazem a transição para o mecanismo de vizinhos próximos em $300^{\circ} \mathrm{C}$ e $220^{\circ} \mathrm{C}$, respectivamente. 
Figura 5. Gráfico da energia de ativação em função da temperatura para as amostras estudadas.

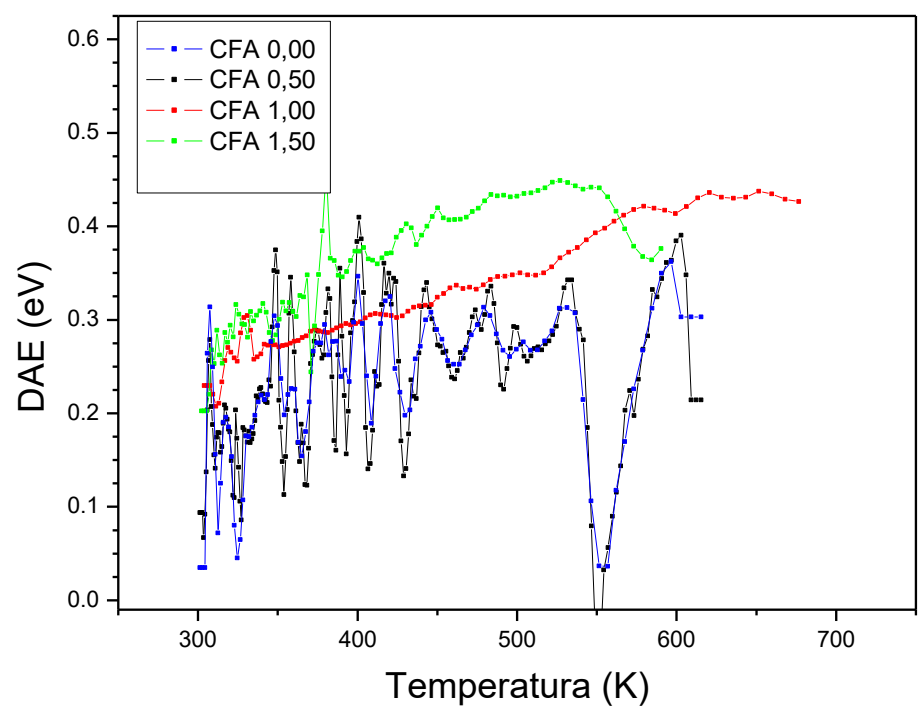

Fonte: Autores (2021).

\section{Conclusão}

A resistividade da amostra diminui com o aumento da temperatura, mostrando um comportamento de um material semicondutor com resistividade entre $10^{4}-10^{5}$ ( ohm $\mathrm{m}$ ). O aumento da resistividade do material com o aumento do teor de alumínio ocorre devido à sua propriedade condutora. Observou-se que aumentando a porcentagem do elemento alumínio, ocorre um aumento do tamanho de cristalito, até que a concentração de alumínio se iguale a de oxido de cobre, a partir da diminuição do oxido de ferro para valores menores do que a concentração do alumínio, o tamanho do cristalito foi diminuindo, atingindo seu valor mínimo para uma baixíssima porcentagem do elemento oxido de ferro.

Os resultados mostraram que a magnetização de saturação máxima foi obtida para a ferrita de $\mathrm{CuFe}_{2} \mathrm{O}_{4}$ em comparação com as outras amostras da série. O aumento da dopagem de alumínio gera uma significativa diminuição da magnetização de saturação e remanência, devido ao fato de o alumínio ser um material paramagnético, enquanto se observa um aumento da coercividade no material.

\section{Agradecimentos}

Os autores agradecem à FAPEMIG, CAPES, UNIFEI e CNPq pelos apoios financeiros.

\section{Referências}

Chae, K. P., Choi, W. O., Lee, J.-G., Kang, B.-S., \& Choi, S. H. (2013). Crystallographic and Magnetic Properties of Nickel Substituted Manganese Ferrites Synthesized by Sol-gel Method. Journal of Magnetics, 18(1), 21-25. https://doi.org/10.4283/jmag.2013.18.1.021

Dunitz, J. D., \& Orgel, L. E. (1957). Electronic properties of transition-metal oxides-I. Journal of Physics and Chemistry of Solids, 3(1-2), 20-29. https://doi.org/10.1016/0022-3697(57)90043-4

Gabal, M. A., Abdel-Daiem, A. M., Al Angari, Y. M., \& Ismail, I. M. (2013). Influence of Al-substitution on structural, electrical and magnetic properties of $\mathrm{Mn}-\mathrm{Zn}$ ferrites nanopowders prepared via the sol-gel auto-combustion method. Polyhedron, 57, 105-111. https://doi.org/10.1016/j.poly.2013.04.027

Jonker, G. H. (1959). Analysis of the semiconducting properties of cobalt ferrite. Journal of Physics and Chemistry of Solids, 9(2), 165-175. https://doi.org/10.1016/0022-3697(59)90206-9

Mathew, D. S., \& Juang, R.-S. (2007). An overview of the structure and magnetism of spinel ferrite nanoparticles and their synthesis in microemulsions. Chemical Engineering Journal, 129(1-3), 51-65. https://doi.org/10.1016/j.cej.2006.11.001 
Oliveira, V. D. de, Rubinger, R. M., Silva, M. R. da, Oliveira, A. F., Rodrigues, G., \& Ribeiro, V. A. dos S. (2016). Magnetic and Electrical Properties of MnxCu1-xFe2O4 Ferrite. Materials Research, 19(4), 786-790. https://doi.org/10.1590/1980-5373-mr-2015-0511

Parfenov, V. V., \& Nazipov, R. A. (2002). Inorganic Materials, 38(1), 78-82. https://doi.org/10.1023/a:1013615930587

Raghavender, A. T., Shirsath, S. E., Pajic, D., Zadro, K., Milekovic, T., Jadhav, K. M., \& Kumar, K. V. (2012). Effect of Al doping on the cation distribution in copper ferrite nanoparticles and their structural and magnetic properties. Journal of the Korean Physical Society, 61(4), 568-574. https://doi.org/10.3938/jkps.61.568

Raghavender, A. T., Shirsath, S. E., Pajic, D., Zadro, K., Milekovic, T., Jadhav, K. M., Kumar, K. V. (2020). Effect of Al Doping on the Cation Distribution in Copper Ferrite Nanoparticles and Their Structural and Magnetic Properties. Journal of the Korean Physical Society, 61 (4), 568- 574. 10.3938/jkps.61.568

Ribeiro, L. H., Oliveira, A. F., \& Rubinger, R. M. (2021). Instrumentação para medidas de mobilidade eletrônica e concentração de portadores em amostras semicondutoras, pelo método de van der Pauw. Research, Society and Development, 10(6), e41310615229. https://doi.org/10.33448/rsd-v10i6.15229

Ribeiro, V. A. dos S., Pereira, A. C., Oliveira, A. F., Mendonça, C. de S. P., \& Silva, M. R. da. (2016). Avaliação da microestrutura e das propriedades magnéticas de ferrita de cobre dopada com chumbo sinterizada com fase líquida. Matéria (Rio de Janeiro), 21(2), 330-341. https://doi.org/10.1590/s1517707620160002.0032

Ribeiro, V. A. dos S., Rubinger, R. M., Oliveira, A. F., Mendonça, C. S. P., \& Silva, M. R. da. (2016). Magnetic properties and potential barrier between crystallites model of MgGa2-xFexO4 ceramics. Cerâmica, 62(364), 365-369. https://doi.org/10.1590/0366-69132016623642006

Rubinger, C. P. L., Costa, L. C., Faez, R., Martins, C. R., \& Rubinger, R. M. (2009). Hopping conduction on PAni/PSS blends. Synthetic Metals, 159(5-6), 523-527. https://doi.org/10.1016/j.synthmet.2008.11.012

Rubinger, R. M., Ribeiro, G. M., Oliveira, A. G. de, Albuquerque, H. A., Silva, R. L. da, Rubinger, C. P. L., Rodrigues, W. N., \& Moreira, M. V. B. (2006). Temperature-dependent activation energy and variable range hopping in semi-insulating GaAs. Semiconductor Science and Technology, 21(12), 1681-1685. https://doi.org/10.1088/0268-1242/21/12/030

Sattar, A. A. (2004). Composition dependence of some physical, magnetic and electrical properties of Ga substituted Mn-ferrites. Journal of Materials Science, 39(2), 451-455. https://doi.org/10.1023/b:jmsc.0000011497.30763.bc

Skołyszewska, B., Tokarz, W., Przybylski, K., \& Kạkol, Z. (2003). Preparation and magnetic properties of MgZn and MnZn ferrites. Physica C: Superconductivity, 387(1-2), 290-294. https://doi.org/10.1016/s0921-4534(03)00696-8

Smit, J. and Wijn, H.P.J. (1959) Ferrites. Philips Technical Library, Eindhoven, 150.

Sugimoto, m. (1977). Cubic-tetragonal transformation and magnetic properties in copper ferrites with excess Fe2O3. Le Journal de Physique Colloques, 38(C1), C1-117-C1-120. https://doi.org/10.1051/jphyscol:1977122

Šutka, A., \& Gross, K. A. (2016). Spinel ferrite oxide semiconductor gas sensors. Sensors and Actuators B: Chemical, 222, 95-105. https://doi.org/10.1016/j.snb.2015.08.027

Surashe, V. K., Mahale, V., Keche, A. P., Alange, R. C., Aghav, P. C., Dorik, R. G. (2020). Structural and electrical properties of copper ferrite (CuFe2O4) NPs. Journal of Physics: Conference Series. 1644 (2020), 1-7. https://doi:10.1088/1742-6596/1644/1/012025

Tanaka, T., Chiba, M., Okimura, H., \& Koizumi, Y. (1997). Jahn-Teller Effect of Cu-Ferrite Films by Solid Reaction. Le Journal de Physique IV, 07(C1), C1501-C1-502. https://doi.org/10.1051/jp4:19971205

Ribeiro, V. A. S. R., Oliveira, A. F., Rubinger, R. M., Mendonça, C. S. P., Oliveira, V. D., Silva, M. R. (2019). Electrical and structural characterization of lead and copper ceramics. Tecnol. metal. mater. min., 16 (2), 284-289. 